\title{
Inflammation and the Silent Sequelae of Stroke
}

\author{
Kyra J. Becker ${ }^{1}$
}

Published online: 20 June 2016

(C) The American Society for Experimental NeuroTherapeutics, Inc. 2016

\begin{abstract}
Depression and fatigue are common after stroke and negatively impact the quality of life of stroke survivors. The biological bases of these symptoms are unknown, but an abundance of data point to a role for inflammation. This review highlights evidence supporting the contribution of inflammation to poststroke depression and poststroke fatigue. Potential treatments for poststroke depression and poststroke fatigue are explored, with a special emphasis on those that modulate the immune response.
\end{abstract}

Keywords Depression · Fatigue $\cdot$ Inflammation $\cdot$ Stroke

\section{Introduction}

Stroke affects approximately 795,000 persons each year in the USA and is the leading cause of permanent adult disability [1]. The physical consequences of stroke are generally obvious to an observer, but there are less perceptible consequences of stroke that may be more incapacitating than the physical disabilities. Poststroke depression (PSD) and poststroke fatigue (PSF) are both common and interfere with a person's ability to reintegrate into daily activities, even in patients with no apparent neurological deficits. Despite the high prevalence of PSD and PSF, the biological underpinnings of these symptoms are unknown. Based on data regarding depression and fatigue in other disease processes, as well as emerging data for stroke, it is possible that the genesis of PSD and PSF might be related to dysregulation of the immune response after stroke.

Kyra J. Becker

kjb@uw.edu

1 University of Washington School of Medicine, Seattle, WA, USA
This paper will review the importance of PSD and PSF, explore the literature that supports an immunological connection to these symptoms, and speculate about the development of potential therapies.

\section{PSD and PSF are Prevalent}

At least one-third of patients who suffer stroke report depression in the poststroke period $[2,3]$. These estimates vary based on the population studied and how depression was defined. Most studies of PSD are cross-sectional; the longitudinal studies that have been done tend to be small and have limited follow-up [4]. Fatigue, defined as a feeling of weariness, tiredness, or lack of energy, affects up to three-quarters of patients who suffer stroke [5-12]. Similar to PSD, most studies of PSF are cross-sectional in design and do not adequately address the natural history of PSF [13].

Fatigue is a manifestation of depression, but multiple lines of evidence suggest that fatigue and depression are distinct clinical entities. First, PSF is more prevalent than PSD, meaning that there is a subset of patients with stroke who develop fatigue without being depressed. For instance, in a study of patients who were at least a year poststroke, only $38 \%$ of the patients with fatigue were depressed [9]. Second, effective treatment of PSD by pharmacologic means does not eliminate PSF $[14,15]$. And, finally, fatigue is more common in patients with stroke who have completely recovered as compared with patients with transient cerebral ischemia (without infarction), despite similar levels of depression and anxiety [16]. This observation suggest that tissue injury might set into motion biochemical changes that lead to fatigue but that anxiety and depression are more related to the psychological aspects of the event. The overlap between PSD and PSF is depicted in Fig. 1. 


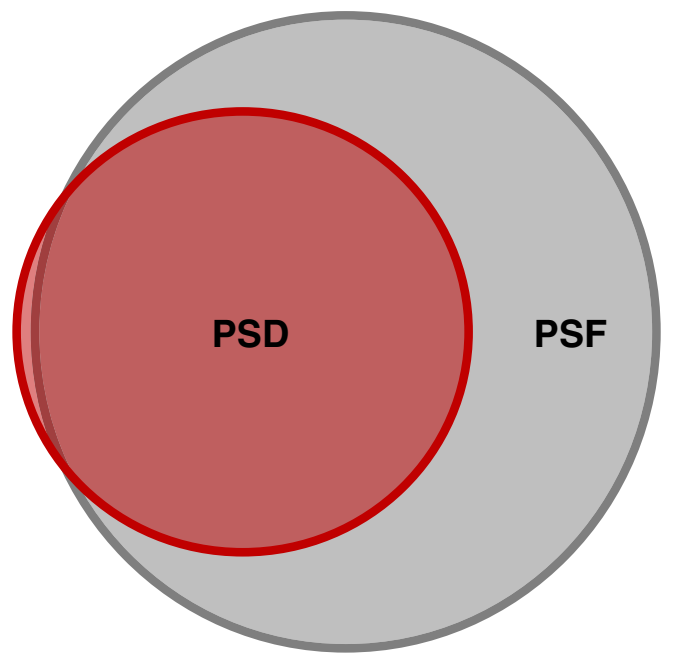

Fig. 1 There is overlap between poststroke depression (PSD) and poststroke fatigue (PSF); most patients with PSD have PSF, but not all patients with PSF have PSD

\section{PSD and PSF Impair Recovery and Decrease Quality of Life}

PSD has been linked to decreased quality of life, increased disability, and increased mortality [4, 17-19]. In addition, there is an increase in suicidal ideation and suicide after stroke, especially among those with PSD [20,21]. Studies also show that individuals with PSD experience a shorter interval to recurrent stroke [22]. And even in patients with minor stroke, PSD is independently associated with disability and poor physical and mental health 1 year after stroke [19].

PSF interferes with physical therapy, and patients identify fatigue as one of the major obstacles to their rehabilitation [23, 24]. The consequences of PSF are further illustrated by the fact that patients who complain of fatigue are more likely to be dependent for their activities of daily living and are more likely to require institutional care; mortality is also higher in patients with PSF [11, 25-27]. Among patients with mild stroke, fatigue is the most common complaint and is identified as the major issue restricting their life style [7, 10]. It is thus not surprising that PSF is more predictive of a lower health-related quality of life after stroke than actual neurological dysfunction [28]. Fatigue also prevents individuals who have experienced otherwise good stroke recovery from returning to work, which means that there are economic, as well as psychological implications to PSF $[29,30]$.

\section{Assessing PSD and PSF}

Studies of PSD use a variety of different rating scales to assess for depressive symptoms, and the distinction between depressive symptoms and a depressive disorder is often not clear [31]. Further, the cut-off points for defining depressive symptoms using individual scales tend to differ from study to study [31]. A meta-analysis suggests that while some of commonly used scales are helpful in screening for PSD, none are satisfactory for case finding [32].

Similar to the situation for PSD, evaluation of the PSF literature quickly demonstrates that there is no consensus regarding the use of fatigue scales in patients with stroke-multiple different scales are used by multiple different research groups. Comparison of the various fatigue scales in patients with stroke shows that several are valid and feasible [33], yet none have been agreed upon as a standard for studies of PSF. A case definition has been proposed, however, and it requires that "Over the past month, there has been at least a 2-week period when the patient has experienced fatigue, a lack of energy, or an increased need to rest every day or nearly every day. This fatigue has led to difficulty taking part in everyday activities" [34].

\section{Risk Factors for PSD and PSF}

Many studies suggest that PSD is associated with a history of prestroke depression. PSD also appears to be related to the degree of neurological disability and functional impairment, as well as to the social support network, socioeconomic status, and education $[35,36]$. Identified risk factors for PSF depend upon the study in question, but depression, female sex, and European ethnicity are often implicated [12]. A relationship between age and PSF is unclear given that some studies show PSF to be more common in younger patients and others show it to be more common in older patients $[12,13]$.

\section{The Contribution of Inflammation to Depression and Fatigue}

A role for inflammation in the genesis of both depression and fatigue is implicated by several lines of evidence. First, depressive-like behavior is common in animals subjected to inflammatory insults $[37,38]$. Second, mood disturbance is common after infection [39]. In the Third National Health and Nutrition Examination Survey (NHANES III), major depression was associated with higher levels of $\mathrm{C}$-reactive protein (CRP), especially in men [40]. Finally, patients treated with proinflammatory cytokines often develop depression. Treatment with interferon- $\gamma$, in particular, is associated with depression [41].

A similar body of evidence exists for the link between inflammation and fatigue. First, it is a common symptom in patients with immune-mediated diseases [42-46]. Second, 
the perception of fatigue is common among otherwise healthy individuals who suffer infectious illnesses [47, 48]. And, as with depression, the administration of proinflammatory cytokines [e.g., interleukin (IL)-6] is associated with the perception of fatigue [49]. Further, treatment with cytokine antagonists is associated with a decrease in fatigue-administration of exogenous IL-1 ra (anakinra) decreases fatigue in patients with Sjögren's syndrome [50], and the tumor necrosis factor (TNF)- $\alpha$ receptor antagonist infliximab and the IL-6 receptor antagonist toclizumab reduce fatigue in patients with cancer and autoimmune diseases [51-55].

\section{Inflammation and the Biological Basis of PSD}

Most studies that have looked for biological markers of PSD are small and have serious methodological issues. Given that inflammation is common after stroke, inflammation is linked to depression, and depression is common after stroke, there seems as if there should be a link between PSD and inflammation [56]. Unfortunately, the poor quality of the studies done to date do not allow for a definitive connection between PSD and inflammation to be made. In some studies, early markers of inflammation like CRP [57], ferritin [58], and neopterin [59] were associated with the later development of PSD. High serum levels of leptin at days 7 and 30 after stroke have also shown an association with later development of PSD [60]. And low levels of vitamin $\mathrm{D}$, which is important for immunoregulation, have been linked to PSD [61]. Finally, elevated plasma glutamate levels at admission are associated with PSD [62]. Glutamate is an excitatory amino acid which is neurotoxic at high concentrations, but glutamate also possesses inflammatory potential, with glutamate receptors playing a role in immune-mediated diseases of the nervous system [63, 64].

In most studies, psychosocial factors are predictive of PSD $[35,36]$. Epigenetics are invoked as a link between psychosocial stressors and the biology that mediates disease onset or outcome [65]. In animal studies, social isolation after stroke leads to behaviors consistent with PSD and is associated with increased serum IL-6 [66].

\section{Inflammation and the Biological Basis of PSF}

There are limited data supporting a role for inflammation in PSF. Similar to the studies for PSD, those studies that have looked for biological markers of PSF are small and have methodological issues. The studies that have been done show a link between PSF and increased IL-1 $\beta$, decreased IL-1ra, and decreased IL-9 at stroke onset $[67,68]$. The data, however, were not controlled for initial stroke severity, age, or other important covariates. More fundamentally, however, is that for a cytokine to mediate fatigue, that cytokine should be measured contemporaneously with the fatigue assessment instead at the time of the initial stroke. When assessed simultaneously, CRP appears to correlate with PSF in some studies [69] but not in others [70].

In a prospective study of patients with ischemic stroke, we assessed biomarkers of inflammation over 1 year after stroke. Patients with severe stroke (National Institutes of health Stroke Scale Score $\geq 17$ ), had elevations in CRP, IL-6, IL1ra, and high mobility group box-1 for many months after stroke onset, and in some instances to at least a year (Fig. 2). In a subset of these patients, fatigue was assessed at 1 month, 3 months, and 1 year after stroke using the Fatigue Assessment Scale (FAS). The FAS has been validated in healthy cohorts [71]. FAS scores of 10-21 indicate that the patient is "not tired", scores of 22-34 indicate that the patient is "tired", and scores of 35-50 indicate that the patient is "very tired" [72]. We evaluated the relationship between the biomarkers of inflammation shown in Fig. 2, as well as other potential biomarkers, and being "tired" (FAS $\geq 22$ ) in the year after stroke (Table 1). After controlling for stroke severity and the time at which fatigue was assessed, CRP, cortisol, and vascular cell adhesion molecule-1 were predictive of fatigue. After controlling for "feeling sad", only CRP and vascular cell adhesion molecule-1 remained significant. In this same cohort of patients, we found that single nucleotide polymorphisms (SNPs) in genes that regulate inflammation were also associated with PSF [73]. Specifically, we found that PSF was associated with the C allele of ILIRN rs4251961, which is commonly associated with a decrease in IL-1ra and an increase in proinflammatory cytokines like IL-1 $\beta$ and CRP $[74,75]$. Further, we found that functional polymorphisms in Toll-like receptor 4 (TLR4) that render it less responsive to its ligands were associated with less fatigue [73]. These data are shown graphically in Fig. 3.

In attempt to better understand the biological basis of PSD and PSF, we evaluated the behavior and spontaneous activity in Lewis and Sprague-Dawley rats 1 month after stroke. Lewis rats are prone to inflammatory and autoimmune disorders, while Sprague-Dawley rats are more resistant to such diseases [76]. Following stroke, Lewis rats developed "learned helplessness", which is a proxy for depression [77]. SpragueDawley rats, however, evidenced "fatigue-like" behavior with a decrease in the velocity of movement; this strain did not develop behavior consistent with depression [77]. In this study, systemic levels of IL- $1 \alpha$ correlated with "learned helplessness" and were higher in Lewis rats. Lower levels of IL$1 \mathrm{ra}$, however, were associated with a decrease in the velocity of movement and the distance moved [77]. These data show that differences in rat strains can be capitalized upon to study the origins of PSD and PSF, and that the inflammatory milieu 

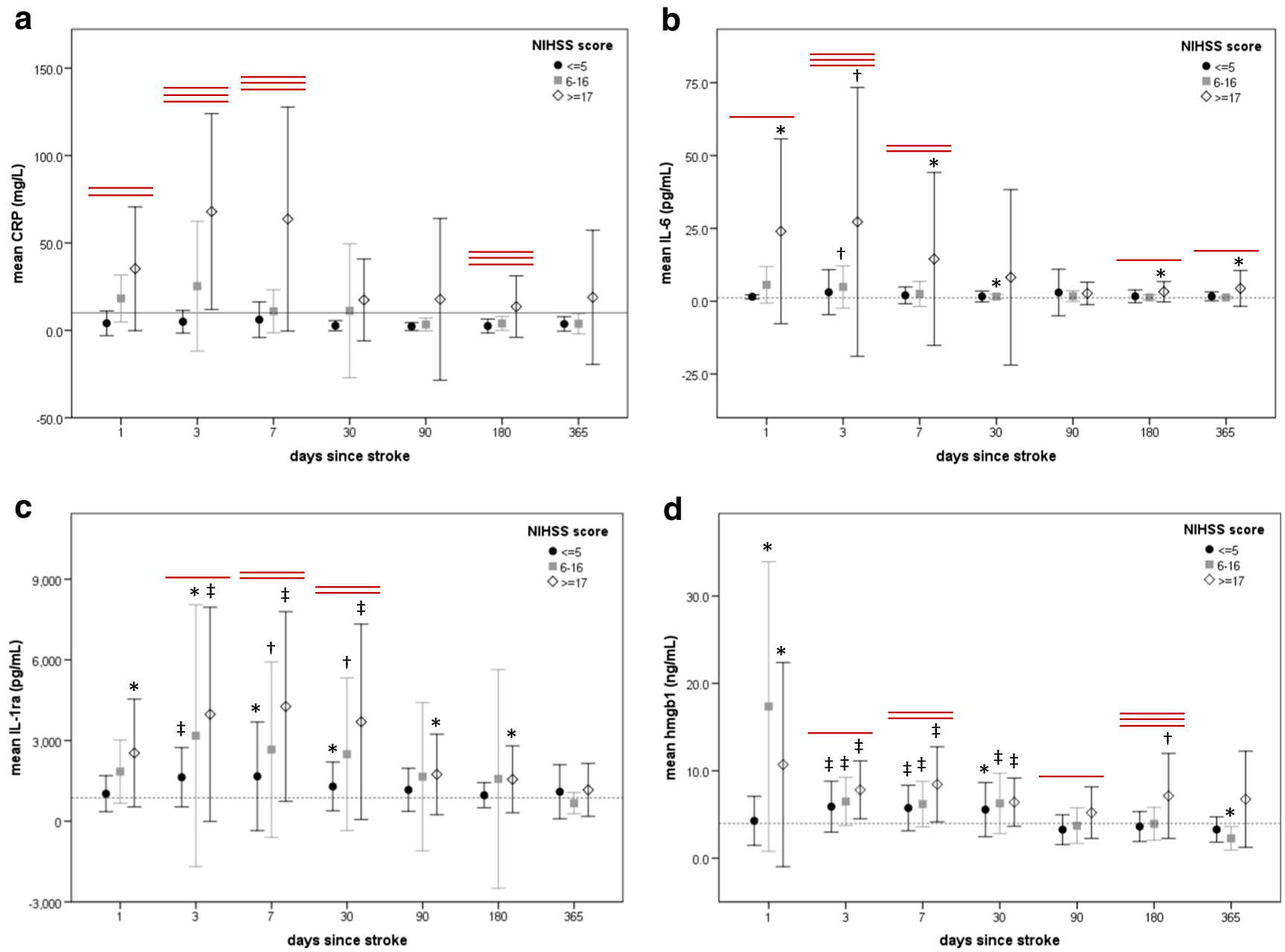

Fig. 2 There is a prolonged inflammatory response after stroke, which is most notable in patients with severe stroke [National Institutes of health Stroke Scale Score (NIHSSS) $\geq 17]$. Compared with patients with less severe stroke, (a) C-reactive protein (CRP), (b) interleukin (IL)-6, and (d) high mobility group box-1 (HMGB-1) are still elevated at 180 days after stroke. Compared with healthy controls, IL-6, (c) IL-1ra, and HMGB-1 are elevated to at least 180 days after stroke. Data are shown as the mean with error bars representing the SD. The dotted black horizontal lines in

in these strains likely affects the propensity to develop behaviors consistent with depression and fatigue.

Finally, neurochemicals, including glutamate, have also been implicated in the genesis of fatigue [78]. In a small clinical study, plasma glutamate levels correlated with the degree of fatigue in patients with minor stroke [79]. While stroke itself can lead to dynamic alterations in the metabolism of neurotransmitters (e.g., glutamate), inflammation also leads to major changes in the metabolism of these neurochemicals [80-82]. Cytokines (like IFN) alter the metabolism of monoamines (including dopamine and serotonin) and may contribute to fatigue [83, 84]. Based on these kinds of data, it appears that all of the major potential causes of fatigue following stroke are therefore intimately related and may share a common denominator in the immune system [85-88].

(b-d) represent the mean value in control patients; the solid horizontal in (a) represents the upper limit of normal for CRP in our laboratory. Differences among stroke patients are represented by horizontal red lines; 1 line indicates $p \leq 0.05,2$ lines indicate $p \leq 0.01$, and 3 horizontal red lines indicate $p \leq 0.001$. Differences between each tertile of stroke severity and control patients are denoted by $* p \leq 0.05,{ }^{\dagger} p \leq 0.01$, or ${ }^{\ddagger} p \leq 0.001$

\section{Therapeutic Approaches to the Treatment of Depression: A Focus on Inflammation}

One of the most common pharmacological treatments for depression is modulation of 5-hydroxytryptophan through the use of selective serotonin reuptake inhibitors (SSRIs). There are ample data that show that this class of drugs also modulates inflammation [89]. For instance, treatment with SSRIs decrease CRP in patients with depression [90]. And in experimental models, SSRIs attenuate lipopolysaccharide-induced inflammation [91]. A variety of more traditional antiinflammatory therapies has also been used for the treatment of depression, including aspirin and other nonsteroidal antiinflammatory drugs, statins, and cytokine inhibitors like etanercept (which blocks TNF) [92-95]. Agomelatine is a 
Table 1 Biomarkers of poststroke fatigue (Fatigue Assessment Score $\geq 22$ ) from 1 month to 1 year after ischemic stroke

\begin{tabular}{|c|c|c|c|c|c|c|c|c|}
\hline & \multicolumn{2}{|l|}{ Uncontrolled } & \multicolumn{2}{|l|}{ Controlled for NIHSSS } & \multicolumn{2}{|l|}{$\begin{array}{l}\text { Controlled for NIHSSS } \\
\text { and time point }\end{array}$} & \multicolumn{2}{|l|}{$\begin{array}{l}\text { Controlled for NIHSSS, } \\
\text { time point, and "feeling } \\
\text { sad" }\end{array}$} \\
\hline & OR $(95 \% \mathrm{CI})$ & $p$ & OR $(95 \% \mathrm{CI})$ & $p$ & OR $(95 \% \mathrm{CI})$ & $p$ & OR $(95 \% \mathrm{CI})$ & $p$ \\
\hline \multicolumn{9}{|l|}{ Inflammatory biomarkers } \\
\hline CRP (per 10 mg/l) & $5.42(1.30-22.58)$ & 0.02 & $8.74(1.81-42.24)$ & 0.007 & $8.72(1.80-42.26)$ & 0.007 & $7.38(1.39-2.26)$ & 0.02 \\
\hline $\mathrm{TNF}-\alpha($ per pg/ml) & $1.26(0.92-1.71)$ & 0.15 & $1.34(0.95-1.90)$ & 0.09 & $1.35(0.95-1.92)$ & 0.09 & $1.33(0.90-1.98)$ & 0.16 \\
\hline IL-6 (per pg/ml) & $1.06(0.95-1.20)$ & NS & $1.09(0.95-1.25)$ & NS & $1.09(0.95-1.25)$ & NS & $1.10(0.96-1.26)$ & 0.17 \\
\hline IL1ra (per 100 pg/ml) & $1.03(0.99-1.08)$ & 0.13 & $1.04(0.99-1.09)$ & 0.11 & $1.04(0.99-1.09)$ & 0.11 & $1.04(0.99-1.10)$ & 0.14 \\
\hline HMGB-1 (per ng/ml) & $1.02(0.86-1.21)$ & NS & $1.01(0.84-1.21)$ & NS & $1.08(0.88-1.32)$ & NS & $1.16(0.91-1.46)$ & NS \\
\hline IL-10 (per pg/ml) & $23.40(0.51-1073.23)$ & NS & $34.68(0.76-1589.81)$ & 0.07 & $33.91(0.72-1599.65)$ & 0.07 & $32.96(0.42-2619.50)$ & 0.12 \\
\hline \multicolumn{9}{|l|}{ Endocrine biomarkers } \\
\hline Cortisol (per $\mu \mathrm{g} / \mathrm{dl})$ & $1.21(1.04-1.42)$ & 0.02 & $1.19(1.01-1.39)$ & 0.04 & $1.19(1.01-1.39)$ & 0.04 & $1.15(0.96-1.37)$ & 0.13 \\
\hline ACTH (per pg/ml) & $0.98(0.94-1.02)$ & NS & $0.99(0.95-1.03)$ & NS & $0.99(0.94-1.03)$ & NS & $(0.93-1.03)$ & NS \\
\hline$\alpha$-MSH (per pg/ml) & $1.02(0.99-1.06)$ & 0.12 & $1.03(1.00-1.07)$ & 0.07 & $1.04(1.00-1.07)$ & 0.05 & $1.03(0.99-1.06)$ & 0.15 \\
\hline \multicolumn{9}{|l|}{ Adhesion molecules } \\
\hline VCAM-1 (per 100 pg/ml) & $0.85(0.72-0.99)$ & 0.04 & $0.84(0.72-1.00)$ & 0.04 & $0.84(0.72-1.00)$ & 0.04 & $0.83(0.70-0.98)$ & 0.03 \\
\hline ICAM-1 (per 100 pg/ml) & $1.00(0.51-1.97)$ & NS & $0.84(0.40-1.78)$ & NS & $0.84(0.40-1.77)$ & NS & $0.69(0.30-1.60)$ & NS \\
\hline FKN (per 100 pg/ml) & $1.02(0.78-1.34)$ & NS & $1.08(0.81-1.43)$ & NS & $1.08(0.81-1.44)$ & NS & $0.96(0.70-1.33)$ & NS \\
\hline
\end{tabular}

NIHSSS = National Institutes of Health Stroke Scale Score; $\mathrm{OR}=$ odds ratio; $\mathrm{CI}=$ confidence interval; $\mathrm{CRP}=\mathrm{C}$-reactive protein; $\mathrm{TNF}=$ tumor necrosis factor; IL = interleukin; NS = nonsignificant; HMGB = high mobility group box; $\mathrm{ACTH}=$ adrenorcorticotropic hormone; $\mathrm{MSH}=$ melanocytestimulating hormone; VCAM = vascular cell adhesion molecule; ICAM = intercellular adhesion molecule; FKN = fractalkine

member of a novel class of new antidepressants which are melatonin agonists; it has been approved by the European Union, after being shown to have similar efficacy to SSRIs for the treatment of depression [96, 97]. Melatonin agonists have numerous effects on the immune system, most of which serve to limit inflammation [98]. Finally, nonpharmacologic

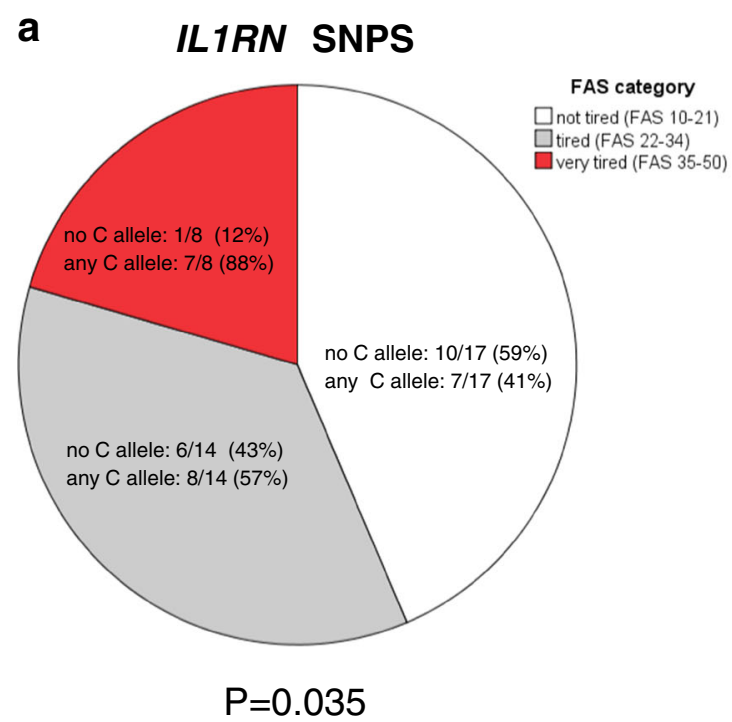

Fig. 3 Poststroke fatigue is more prevalent in those with the $\mathrm{C}$ allele of $\operatorname{ILIRN~(a),~which~is~generally~associated~with~increased~inflammation~}$ $[74,75]$, and less prevalent in those with single nucleotide approaches are also used to treat depression; these approaches include vagal nerve stimulation and exercise. Vagal nerve stimulation, approved for the treatment of resistant depression, leads to immunomodulation in animals [99]. Exercise also appears to decrease systemic markers of inflammation [100, 101] and lessen depression [102].

\section{b}

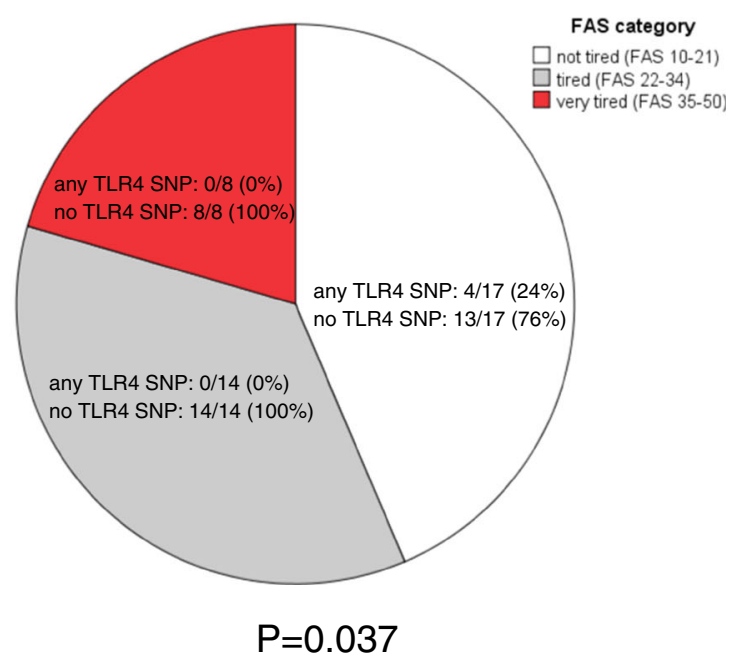

polymorphisms (SNPs) in TLR4 (b) that render the Toll-like receptor less sensitive to its agonists. FAS Fatigue Assessment Scale 
If inflammation contributes to depression, the increased inflammatory state following stroke would suggest that antiinflammatory therapies may be particular beneficial in treating PSD. Studies show that traditional antidepressants, like the SSRIs, have some benefit for the treatment of PSD [14, 15, 103-105]. The quality of these studies, however, has been suboptimal and the evidence is not yet compelling enough to suggest that antidepressants be prescribed to all patients with PSD [106]. Non-SSRI antidepressants have not been widely studied in stroke, but drugs like agomelatine may be particularly attractive given that melatonin decreases central nervous system inflammation [107] and improves outcome in experimental models of cerebral ischemia [108, 109]. Additional evidence for the role of inflammation in the genesis of PSD and as a viable target for the treatment of PSD includes the fact that treatment with pioglitazone, as opposed to metformin, decreases PSD in patients with diabetes [110]. Peroxisome proliferator-activated receptor- $\gamma$ agonists like pioglitazone have both anti-inflammatory and neuroprotective properties $[111,112]$. And in a recent trial of secondary stroke prevention, pioglitazone decreased CRP in patients with insulin resistance [113]. Exercise, which is known to decrease CRP, has also been shown to be of benefit in PSD [114, 115]. And perhaps most exciting is that fact that treatment with natalizumab, an antibody to the $\alpha 4$ component of very late antigen-4 expressed on monocytes and lymphocytes, was associated with less depression after stroke [116]. This finding suggests that the inhibition of the immune response after stroke can prevent PSD.

\section{Therapeutic Approaches to the Treatment of Fatigue: A Focus on Inflammation}

Fatigue is common in patients with cancer and in patients with autoimmune disease. Inhibiting proinflammatory cytokines in these patients appears to lessen fatigue. Treatment with exogenous IL-1ra (anakinra), for instance, decreases fatigue in patients with Sjögren's syndrome [50]. Similarly, the TNF- $\alpha$ receptor antagonist infliximab and the IL-6 receptor antagonist tocilizumab reduce fatigue in patients with cancer [51] and a variety of autoimmune diseases [52-55]. Fatigue is a particularly prevalent problem for patients with multiple sclerosis (MS) [117]. While a number of different drugs are used to treat MS-related fatigue, the data providing support for pharmacologic interventions are, at best, weak [118]. Exercise therapy may help treat fatigue in patients with MS, but the quality of most of these trials is limited [119]. Predicated on the belief that MS-related fatigue may be related to inflammation, studies were undertaken with aspirin; this nonsteroidal anti-inflammatory drug was shown to improve fatigue in patients with MS in 2 different clinical studies $[120,121]$.
Given that there is not a clearly defined etiology for PSF, there are no rationally informed interventions. To date, very few trials have been done to address therapies for PSF. In fact, the most recent Cochrane review concluded that "there is insufficient evidence available to guide the management of fatigue after stroke" [122]. What we do know is that antidepressants do not seem to improve PSD [14]. Modafinil, which is approved for the treatment of fatigue in shift workers and patients with MS, failed to improve the Multi-dimensional Fatigue Inventory-20 score in a relatively small randomized controlled trial of patients with stroke, although it did have an effect on some secondary endpoints [123]. Cognitive therapy with graded activity training, however, was associated with a decrease in PSF [124]. (As mentioned previously, exercise and fitness training lead to a reduction in circulating proinflammatory cytokines [125-127].) All of these intervention trials are small and the results need to be confirmed.

If dysregulation of the immune response is an important contributing factor to PSF, interventions that lessen inflammation would be appropriate treatment strategies. Based on the studies that show a decrease in MS related fatigue with aspirin $[120,121]$, and as aspirin is prescribed to virtually all patients with ischemic stroke (who do not need to be anticoagulated), it would be reasonable to consider aspirin, albeit at a higher dose than is normally used for secondary stroke prevention, as a treatment for PSF. For example, at doses of $300 \mathrm{mg}$ a day, aspirin decreases plasma concentrations of CRP, TNF- $\alpha$, and IL-6 [128, 129]. Data regarding the effects of even higher doses of aspirin on these biomarkers of inflammation are limited. Other pharmacologic interventions using drugs that modulate the immune response could also be considered to treat PSF. For instance, treatment with natalizumab, which inhibits lymphocytes and monocytes, is associated with decreased fatigue in patients with MS [130]. This same drug is now being explored as a therapy for acute stroke, and assessing PSF in randomized controlled trials that evaluate acute stroke therapeutics may provide insight into the etiology, as well as the treatment, of PSF.

\section{Summary}

PSD and PSF are common sequelae of stroke and are more predictive of quality of life than actual neurological disability. There are data which suggest, but do not prove, a link between systemic inflammation and PSD and PSF. And while there is overlap between PSD and PSF, these symptoms appear to be distinct. It seems very unlikely that a single cytokine or other biological substrate causes either PSD or PSF, but rather it is likely that the overall systemic milieu after stroke that leads to these symptoms. 
Required Author Forms Disclosure forms provided by the authors are available with the online version of this article.

\section{References}

1. Mozaffarian, D., E.J. Benjamin, A.S. Go, et al. Heart Disease and Stroke Statistics-2016 Update: A Report From the American Heart Association. Circulation 2016. 133: p. e38-e360.

2. Gaete, J.M. and J. Bogousslavsky. Post-stroke depression. Expert Rev Neurother 2008. 8: p. 75-92.

3. Staub, F. and J. Bogousslavsky. Post-stroke depression or fatigue. Eur Neurol 2001. 45: p. 3-5.

4. Ayerbe, L., S. Ayis, C.D. Wolfe, and A.G. Rudd. Natural history, predictors and outcomes of depression after stroke: systematic review and meta-analysis. Br J Psychiatry 2013. 202: p. 14-21.

5. Appelros, P. Prevalence and predictors of pain and fatigue after stroke: a population-based study. Int J Rehabil Res 2006. 29: p. 329-333.

6. Choi-Kwon, S., S.W. Han, S.U. Kwon, and J.S. Kim. Poststroke fatigue: characteristics and related factors. Cerebrovasc Dis 2005. 19: p. 84-90.

7. Carlsson, G.E., A. Moller, and C. Blomstrand. Consequences of mild stroke in persons $<75$ years - a 1 -year follow-up. Cerebrovasc Dis 2003. 16: p. 383-388.

8. Schepers, V.P., A.M. Visser-Meily, M. Ketelaar, and E. Lindeman. Poststroke fatigue: course and its relation to personal and strokerelated factors. Arch Phys Med Rehabil 2006. 87: p. 184-188.

9. van der Werf, S.P., H.L. van den Broek, H.W. Anten, and G. Bleijenberg. Experience of severe fatigue long after stroke and its relation to depressive symptoms and disease characteristics. Eur Neurol 2001. 45: p. 28-33.

10. Parks, N.E., G.A. Eskes, G.J. Gubitz, et al. Fatigue impact scale demonstrates greater fatigue in younger stroke survivors. Can J Neurol Sci 2012. 39: p. 619-625.

11. Naess, H., L. Lunde, J. Brogger, and U. Waje-Andreassen. Fatigue among stroke patients on long-term follow-up. The Bergen Stroke Study. J Neurol Sci 2011. 312: p. 138-141.

12. Feigin, V.L., S. Barker-Collo, V. Parag, et al.,Prevalence and predictors of 6-month fatigue in patients with ischemic stroke: a population-based stroke incidence study in Auckland, New Zealand, 2002-2003. Stroke 2012. 43: p. 2604-2609.

13. Snaphaan, L., S. van der Werf, and F.E. de Leeuw. Time course and risk factors of post-stroke fatigue: a prospective cohort study. Eur J Neurol 2010. 18: p. 611-617.

14. Choi-Kwon, S., J. Choi, S.U. Kwon, D.W. Kang, and J.S. Kim. Fluoxetine is not effective in the treatment of post-stroke fatigue: a double-blind, placebo-controlled study. Cerebrovasc Dis 2007. 23: p. 103-108.

15. Karaiskos, D., E. Tzavellas, K. Spengos, S. Vassilopoulou, and T. Paparrigopoulos. Duloxetine versus citalopram and sertraline in the treatment of poststroke depression, anxiety, and fatigue. J Neuropsychiatry Clin Neurosci 2012. 24: p. 349-353.

16. Winward, C., C. Sackley, Z. Metha, and P.M. Rothwell. A population-based study of the prevalence of fatigue after transient ischemic attack and minor stroke. Stroke 2009. 40: p. 757-761.

17. Paolucci, S., C. Gandolfo, L. Provinciali, R. Torta, and V. Toso. The Italian multicenter observational study on post-stroke depression (DESTRO). J Neurol 2006. 253: p. 556-562.

18. Schwab-Malek, S., B. Vatankhah, U. Bogdahn, M. Horn, and H.J. Audebert. Depressive symptoms and quality of life after thrombolysis in stroke: the TEMPiS study. J Neurol 2010. 257: p. $1848-1854$.
19. Shi, Y.Z., Y.T. Xiang, Y. Yang, et al. Depression after minor stroke: the association with disability and quality of life - a 1year follow-up study. Int J Geriatr Psychiatry 2016;31:421-427.

20. Pompili, M., P. Venturini, D.A. Lamis, et al. Suicide in stroke survivors: epidemiology and prevention. Drugs Aging 2015. 32: p. 21-29.

21. Dou, J., J. Tang, C.H. Lu, E.S. Jiang, and P.X. Wang. A study of suicidal ideation in acute ischemic stroke patients. Health Qual Life Outcomes 2015. 13: p. 7.

22. Sibolt, G., S. Curtze, S. Melkas, et al. Post-stroke depression and depression-executive dysfunction syndrome are associated with recurrence of ischaemic stroke. Cerebrovasc Dis 2013. 36: p. 336-343.

23. Morley, W., K. Jackson, and G.E. Mead. Post-stroke fatigue: an important yet neglected symptom. Age Ageing 2005. 34: p. 313.

24. Bendz, M. The first year of rehabilitation after a stroke-from two perspectives. Scand J Caring Sci 2003. 17: p. 215-222.

25. Glader, E.L., B. Stegmayr, and K. Asplund. Poststroke fatigue: a 2-year follow-up study of stroke patients in Sweden. Stroke 2002. 33: p. 1327-1333.

26. Naess, H., L. Lunde, and J. Brogger. The effects of fatigue, pain, and depression on quality of life in ischemic stroke patients: the Bergen Stroke Study. Vasc Health Risk Manag 2012. 8: p. 407413.

27. Mead, G.E., C. Graham, P. Dorman, et al. Fatigue after stroke: baseline predictors and influence on survival. Analysis of data from UK patients recruited in the International Stroke Trial. PLoS One 2011. 6: p. e16988.

28. Naess, H., H.I. Nyland, L. Thomassen, J. Aarseth, and K.M. Myhr. Fatigue at long-term follow-up in young adults with cerebral infarction. Cerebrovasc Dis 2005. 20: p. 245-250.

29. Roding, J., B. Lindstrom, J. Malm, and A. Ohman. Frustrated and invisible-younger stroke patients' experiences of the rehabilitation process. Disabil Rehabil 2003. 25: p. 867-874.

30. Andersen, G., D. Christensen, M. Kirkevold, and S.P. Johnsen. Post-stroke fatigue and return to work: a 2-year follow-up. Acta Neurol Scand 2012. 125: p. 248-253.

31. Kouwenhoven, S.E., M. Kirkevold, K. Engedal, and H.S. Kim. Depression in acute stroke: prevalence, dominant symptoms and associated factors. A systematic literature review. Disabil Rehabil 2011. 33: p. 539-556.

32. Meader, N., T. Moe-Byrne, A. Llewellyn, and A.J. Mitchell. Screening for poststroke major depression: a meta-analysis of diagnostic validity studies. J Neurol Neurosurg Psychiatry 2014;85: 198-206.

33. Mead, G., J. Lynch, C. Greig, et al. Evaluation of fatigue scales in stroke patients. Stroke 2007. 38: p. 2090-2095.

34. Lynch, J., G. Mead, C. Greig, et al. Fatigue after stroke: the development and evaluation of a case definition. J Psychosom Res 2007. 63: p. 539-544.

35. Townend, B.S., S. Whyte, T. Desborough, et al. Longitudinal prevalence and determinants of early mood disorder post-stroke. J Clin Neurosci 2007. 14: p. 429-434.

36. White, J.H., J. Attia, J. Sturm, G. Carter, and P. Magin. Predictors of depression and anxiety in community dwelling stroke survivors: a cohort study. Disabil Rehabil 2014. 36: p. 1975-1982.

37. Fu, X., S.M. Zunich, J.C. O'Connor, et al. Central administration of lipopolysaccharide induces depressive-like behavior in vivo and activates brain indoleamine 2,3 dioxygenase in murine organotypic hippocampal slice cultures. J Neuroinflammation 2010. 7: p. 43

38. Gibney, S.M., B. McGuinness, C. Prendergast, A. Harkin, and T.J. Connor. Poly I:C-induced activation of the immune response is accompanied by depression and anxiety-like behaviours, kynurenine pathway activation and reduced BDNF expression. Brain Behav Immun 2013. 28: p. 170-181. 
39. Gunaratne, P., A.R. Lloyd, and U. Vollmer-Conna. Mood disturbance after infection. Aust N Z J Psychiatry 2013. 47: p. 11521164.

40. Ford, D.E. and T.P. Erlinger, Depression and C-reactive protein in US adults: data from the Third National Health and Nutrition Examination Survey. Arch Intern Med 2004. 164: p. 1010-1014.

41. Udina, M., P. Castellvi, J. Moreno-Espana, et al. Interferoninduced depression in chronic hepatitis $\mathrm{C}$ : a systematic review and meta-analysis. J Clin Psychiatry 2012. 73: p. 1128-1138.

42. Harboe, E., A.B. Tjensvoll, H.K. Vefring, et al. Fatigue in primary Sjogren's syndrome - a link to sickness behaviour in animals? Brain Behav Immun 2009. 23: p. 1104-1108.

43. Newton, J.L. Fatigue in primary biliary cirrhosis. Clin Liver Dis 2008. 12: p. 367-383; ix.

44. Krupp, L.B. and C. Christodoulou. Fatigue in multiple sclerosis. Curr Neurol Neurosci Rep 2001. 1: p. 294-298.

45. Ramsey-Goldman, R. and N. Rothrock, Fatigue in systemic lupus erythematosus and rheumatoid arthritis. PM R 2010. 2: p. 384392

46. Abbas, G., R.A. Jorgensen, and K.D. Lindor. Fatigue in primary biliary cirrhosis. Nat Rev Gastroenterol Hepatol 2010. 7: p. 313-319.

47. Harrison, N.A., L. Brydon, C. Walker, et al. Neural origins of human sickness in interoceptive responses to inflammation. Biol Psychiatry 2009. 66: p. 415-422.

48. Dantzer, R. and K.W. Kelley. Twenty years of research on cytokine-induced sickness behavior. Brain Behav Immun 2007;21:153-160.

49. Tate, J., T. Olencki, J. Finke, et al. Phase I trial of simultaneously administered GM-CSF and IL-6 in patients with renalcell carcinoma: clinical and laboratory effects. Ann Oncol 2001. 12: p. 655-659.

50. Norheim, K.B., E. Harboe, L.G. Goransson, and R. Omdal. Interleukin-1 inhibition and fatigue in primary Sjogren's syndrome-a double blind, randomised clinical trial. PLoS One 2012. 7: p. e30123.

51. Tookman, A.J., C.L. Jones, M. Dewitte, and P.J. Lodge. Fatigue in patients with advanced cancer: a pilot study of an intervention with infliximab. Support Care Cancer 2008;16:1131-1140.

52. Baranauskaite, A., H. Raffayova, N.V. Kungurov, et al. Infliximab plus methotrexate is superior to methotrexate alone in the treatment of psoriatic arthritis in methotrexate-naive patients: the RESPOND study. Ann Rheum Dis 2012. 71: p. 541-548.

53. Matsuyama, M., T. Suzuki, H. Tsuboi, et al. Anti-interleukin-6 receptor antibody (tocilizumab) treatment of multicentric Castleman's disease. Intern Med 2007. 46: p. 771-774.

54. 54. Strand, V., G.R. Burmester, S. Ogale, et al. Improvements in health-related quality of life after treatment with tocilizumab in patients with rheumatoid arthritis refractory to tumour necrosis factor inhibitors: results from the 24-week randomized controlled RADIATE study. Rheumatology (Oxford) 2012. 51: p. 18601869.

55. Burmester, G.R., E. Feist, H. Kellner, et al. Effectiveness and safety of the interleukin 6-receptor antagonist tocilizumab after 4 and 24 weeks in patients with active rheumatoid arthritis: the first phase IIIb real-life study (TAMARA). Ann Rheum Dis 2011. 70: p. 755-759.

56. Pascoe, M.C., S.G. Crewther, L.M. Carey, and D.P. Crewther. Inflammation and depression: why poststroke depression may be the norm and not the exception. Int J Stroke 2011. 6: p. 128-135.

57. Yang, R.R., B.C. Lu, T. Li, et al. The relationship between highsensitivity C-reactive protein at admission and post stroke depression: a 6-month follow-up study. Int J Geriatr Psychiatry 2016. 31: p. 231-239.
58. Zhu, L., B. Han, L. Wang, et al. The association between serum ferritin levels and post-stroke depression. J Affect Disord 2016. 190: p. 98-102.

59. Tang, C.Z., Y.L. Zhang, W.S. Wang, W.G. Li, and J.P. Shi. Elevated serum levels of neopterin at admission predicts depression after acute ischemic stroke: a 6-month follow-up study. Mol Neurobiol 2016;53:3194-3204.

60. Jimenez, I., T. Sobrino, M. Rodriguez-Yanez, et al. High serum levels of leptin are associated with post-stroke depression. Psychol Med 2009. 39: p. 1201-1209.

61. Han, B., Y. Lyu, H. Sun, Y. Wei, and J. He. Low serum levels of vitamin D are associated with post-stroke depression. Eur J Neurol 2015. 22: p. 1269-1274.

62. Cheng, S.Y., Y.D. Zhao, J. Li, et al. Plasma levels of glutamate during stroke is associated with development of post-stroke depression. Psychoneuroendocrinology 2014. 47: p. 126-135.

63. Sulkowski, G., B. Dabrowska-Bouta, M. Chalimoniuk, and L. Struzynska. Effects of antagonists of glutamate receptors on proinflammatory cytokines in the brain cortex of rats subjected to experimental autoimmune encephalomyelitis. J Neuroimmunol 2013. 261: p. 67-76.

64. Bolton, C. and C. Paul, Glutamate receptors in neuroinflammatory demyelinating disease. Mediators Inflamm 2006. 2006: p. 93684.

65. Litzelman, K. and M. Verma. Epigenetic regulation in biopsychosocial pathways. Methods Mol Biol, 2015. 1238: p. 549-567.

66. Verma, R., B.D. Friedler, N.M. Harris, and L.D. McCullough. Pair housing reverses post-stroke depressive behavior in mice. Behav Brain Res 2014. 269: p. 155-163.

67. Ormstad, H., H.C. Aass, K.F. Amthor, N. Lund-Sorensen, and L. Sandvik, Serum cytokine and glucose levels as predictors of poststroke fatigue in acute ischemic stroke patients. J Neurol 2011. 258: p. 670-676.

68. Ormstad, H., H.C. Aass, K.F. Amthor, N. Lund-Sorensen, and L. Sandvik. Serum levels of cytokines, glucose, and hemoglobin as possible predictors of poststroke depression, and association with poststroke fatigue. Int J Neurosci 2012. 122: p. 682-690.

69. McKechnie, F., S. Lewis, and G. Mead. A pilot observational study of the association between fatigue after stroke and Creactive protein. J R Coll Physicians Edinb 2010. 40: p. 9-12.

70. Wu, S., F. Duncan, N.H. Anderson, et al. Exploratory cohort study of associations between serum C-reactive protein and fatigue after stroke. PLoS One 2015. 10: p. e0143784.

71. Michielsen, H.J., J. De Vries, and G.L. Van Heck. Psychometric qualities of a brief self-rated fatigue measure: The Fatigue Assessment Scale. J Psychosom Res 2003. 54: p. 345-352.

72. Michielsen, H.J., M. Drent, T. Peros-Golubicic, and J. De Vries. Fatigue is associated with quality of life in sarcoidosis patients. Chest 2006. 130: p. 989-994.

73. Becker, K., R. Kohen, R. Lee, et al. Poststroke fatigue: hints to a biological mechanism. J Stroke Cerebrovasc Dis 2015. 24: p. 618621.

74. Reiner, A.P., M.M. Wurfel, L.A. Lange, et al. Polymorphisms of the IL1-receptor antagonist gene (IL1RN) are associated with multiple markers of systemic inflammation. Arterioscler Thromb Vasc Biol 2008. 28: p. 1407-1412.

75. Rafiq, S., K. Stevens, A.J. Hurst, et al. Common genetic variation in the gene encoding interleukin-1-receptor antagonist (IL-1RA) is associated with altered circulating IL-1RA levels. Genes Immun 2007. 8: p. 344-351.

76. Hoffman, P.M., J.M. Powers, M.J. Weise, and S.W. Brostoff. Experimental allergic neuritis. I. Rat strain differences in the response to bovine myelin antigens. Brain Res 1980. 195: p. 355-362. 
77. Kunze, A., D. Zierath, O. Drogomiretskiy, and K. Becker. Strain differences in fatigue and depression after experimental stroke. Transl Stroke Res 2014;5:604-611.

78. Ronnback, L. and E. Hansson. On the potential role of glutamate transport in mental fatigue. J Neuroinflammation 2004. 1: p. 22.

79. Syed, A.B., L.M. Castell, A. Ng, C. Winward, and P.M. Rothwell. Plasma glutamate levels predict fatigue after TIA and minor stroke. Cerebrovasc Dis 2007. 23: p. 117.

80. McNally, L., Z. Bhagwagar, and J. Hannestad. Inflammation, glutamate, and glia in depression: a literature review. CNS Spectr 2008. 13: p. 501-510.

81. Fogal, B. and S.J. Hewett, Interleukin-1beta: a bridge between inflammation and excitotoxicity? J Neurochem 2008. 106: p. 123.

82. Vesce, S., D. Rossi, L. Brambilla, and A. Volterra. Glutamate release from astrocytes in physiological conditions and in neurodegenerative disorders characterized by neuroinflammation. Int Rev Neurobiol 2007. 82: p. 57-71.

83. Miller, A.H., S. Ancoli-Israel, J.E. Bower, L. Capuron, and M.R. Irwin. Neuroendocrine-immune mechanisms of behavioral comorbidities in patients with cancer. J Clin Oncol 2008. 26: p. 971-982.

84. Katafuchi, T., T. Kondo, S. Take, and M. Yoshimura. Brain cytokines and the 5-HT system during poly I:C-induced fatigue. Ann N Y Acad Sci 2006. 1088: p. 230-237.

85. Hopkins, S.J. Central nervous system recognition of peripheral inflammation: a neural, hormonal collaboration. Acta Biomed 2007. 78(Suppl. 1): p. 231-247.

86. Leonard, B.E. and A. Myint, Inflammation and depression: is there a causal connection with dementia? Neurotox Res 2006. 10: p. 149-160.

87. Wichers, M. and M. Maes, The psychoneuroimmunopathophysiology of cytokine-induced depression in humans. Int J Neuropsychopharmacol 2002. 5: p. 375-388.

88. Jager, A., S. Sleijfer, and C.C. van der Rijt, The pathogenesis of cancer related fatigue: could increased activity of proinflammatory cytokines be the common denominator? Eur J Cancer 2008. 44: p. 175-181.

89. Walker, F.R. A critical review of the mechanism of action for the selective serotonin reuptake inhibitors: do these drugs possess anti-inflammatory properties and how relevant is this in the treatment of depression? Neuropharmacology 2013. 67: p. 304-317.

90. Chavda, N., N.D. Kantharia, and Jaykaran, Effects of fluoxetine and escitalopram on C-reactive protein in patients of depression. J Pharmacol Pharmacother 2011. 2: p. 11-16.

91. Dong, C., J.C. Zhang, W. Yao, et al. Effects of escitalopram, Rcitalopram, and reboxetine on serum levels of tumor necrosis factor-alpha, interleukin-10, and depression-like behavior in mice after lipopolysaccharide administration. Pharmacol Biochem Behav 2016. 144: p. 7-12.

92. Kohler, O., M.E. Benros, M. Nordentoft, et al. Effect of antiinflammatory treatment on depression, depressive symptoms, and adverse effects: a systematic review and meta-analysis of randomized clinical trials. JAMA Psychiatry 2014. 71: p. 1381-1391.

93. Glaus, J., C.L. Vandeleur, A.M. Lasserre, et al. Aspirin and statin use and the subsequent development of depression in men and women: results from a longitudinal population-based study. J Affect Disord 2015. 182: p. 126-131.

94. Weinberger, J.F., C.L. Raison, D.B. Rye, et al. Inhibition of tumor necrosis factor improves sleep continuity in patients with treatment resistant depression and high inflammation. Brain Behav Immun 2015. 47: p. 193-200.

95. Raison, C.L., R.E. Rutherford, B.J. Woolwine, et al. A randomized controlled trial of the tumor necrosis factor antagonist infliximab for treatment-resistant depression: the role of baseline inflammatory biomarkers. JAMA Psychiatry 2013. 70: p. 31-41.
96. Taylor, D., A. Sparshatt, S. Varma, and O. Olofinjana. Antidepressant efficacy of agomelatine: meta-analysis of published and unpublished studies. BMJ 2014. 348: p. g1888.

97. Guaiana, G., S. Gupta, D. Chiodo, et al. Agomelatine versus other antidepressive agents for major depression. Cochrane Database Syst Rev, 2013. 12: p. CD008851.

98. Radogna, F., M. Diederich, and L. Ghibelli. Melatonin: a pleiotropic molecule regulating inflammation. Biochem Pharmacol 2010. 80: p. 1844-1852.

99. Pavlov, V.A. and K.J. Tracey. Neural circuitry and immunity. Immunol Res 2015. 63: p. 38-57.

100. Lancaster, G.I. and M.A. Febbraio. The immunomodulating role of exercise in metabolic disease. Trends Immunol 2014. 35: p. 262-269.

101. Nimmo, M.A., M. Leggate, J.L. Viana, and J.A. King. The effect of physical activity on mediators of inflammation. Diabetes Obes Metab 2013. 15(Suppl. 3): p. 51-60.

102. Rimer, J., K. Dwan, D.A. Lawlor, et al. Exercise for depression. Cochrane Database Syst Rev 2012. 7: p. CD004366.

103. Fruehwald, S., E. Gatterbauer, P. Rehak, and U. Baumhackl. Early fluoxetine treatment of post-stroke depression-a three-month double-blind placebo-controlled study with an open-label long-term follow up. J Neurol 2003. 250: p. 347-351.

104. Kucukalic, A., A. Bravo-Mehmedbasic, A.D. Kulenovic, and E. Suljic-Mehmedika. Venlafaxine efficacy and tolerability in the treatment of post-stroke depression. Psychiatr Danub 2007. 19: p. 56-60.

105. Chollet, F., J. Tardy, J.F. Albucher, et al. Fluoxetine for motor recovery after acute ischaemic stroke (FLAME): a randomised placebo-controlled trial. Lancet Neurol 2011. 10: p. 123-130.

106. Dwyer Hollender, K., Screening, diagnosis, and treatment of poststroke depression. J Neurosci Nurs 2014. 46: p. 135-141.

107. Esposito, E. and S. Cuzzocrea, Antiinflammatory activity of melatonin in central nervous system. Curr Neuropharmacol 2010. 8: p. 228-242.

108. Lee, M.Y., Y.H. Kuan, H.Y. Chen, et al. Intravenous administration of melatonin reduces the intracerebral cellular inflammatory response following transient focal cerebral ischemia in rats. $\mathrm{J}$ Pineal Res 2007. 42: p. 297-309.

109. Zou, L.Y., S.R. Liu, G. Li, L. Huang, and E.S. Yang, Melatonin reduced volume of cerebral infarct induced by photothrombosis in wild-type mice, not in Cyclooxygenase-1 gene knockout mice. Conf Proc IEEE Eng Med Biol Soc 2004. 7: p. 4748-4750.

110. Hu, Y., H. Xing, X. Dong, et al. Pioglitazone is an effective treatment for patients with post-stroke depression combined with type 2 diabetes mellitus. Exp Ther Med 2015. 10: p. 1109-1114.

111. Wahli, W. and L. Michalik, PPARs at the crossroads of lipid signaling and inflammation. Trends Endocrinol Metab 2012. 23: p. 351-363.

112. Gillespie, W., N. Tyagi, and S.C. Tyagi, Role of PPARgamma, a nuclear hormone receptor in neuroprotection. Indian J Biochem Biophys 2011. 48: p. 73-81.

113. Kernan, W.N., C.M. Viscoli, K.L. Furie, et al. Pioglitazone after ischemic stroke or transient ischemic attack. N Engl J Med 2016;374:1321-1331.

114. Smith, P.S. and M. Thompson. Treadmill training post stroke: are there any secondary benefits? A pilot study. Clin Rehabil 2008. 22: p. 997-1002.

115. Eng, J.J. and B. Reime. Exercise for depressive symptoms in stroke patients: a systematic review and meta-analysis. Clin Rehabil 2014. 28: p. 731-739.

116. Hughes, S. (2016) Could MS Drug Have a Role in Acute Stroke? Medscape Medical News.

117. Patejdl, R., I.K. Penner, T.K. Noack, and U.K. Zettl. Multiple sclerosis and fatigue: a review on the contribution of inflammation 
and immune-mediated neurodegeneration. Autoimmun Rev 2016. 15: p. 210-220.

118. Mucke, M., H. Cuhls, V. Peuckmann-Post, et al. Pharmacological treatments for fatigue associated with palliative care. Cochrane Database Syst Rev 2015. 5: p. CD006788.

119. Heine, M., I. van de Port, M.B. Rietberg, E.E. van Wegen, and G. Kwakkel. Exercise therapy for fatigue in multiple sclerosis. Cochrane Database Syst Rev 2015. 9: p. CD009956.

120. Shaygannejad, V., M. Janghorbani, F. Ashtari, and H. Zakeri. Comparison of the effect of aspirin and amantadine for the treatment of fatigue in multiple sclerosis: a randomized, blinded, crossover study. Neurol Res 2012. 34: p. 854-858.

121. Wingerchuk, D.M., E.E. Benarroch, P.C. O'Brien, et al. A randomized controlled crossover trial of aspirin for fatigue in multiple sclerosis. Neurology 2005. 64: p. 1267-1269.

122. McGeough, E., A. Pollock, L.N. Smith, et al. Interventions for post-stroke fatigue. Cochrane Database Syst Rev 2009: p. CD007030.

123. Poulsen, M.B., B. Damgaard, B. Zerahn, K. Overgaard, and R.S. Rasmussen. Modafinil may alleviate poststroke fatigue: a randomized, placebo-controlled, double-blinded trial. Stroke 2015. 46: p. 3470-3477.

124. Zedlitz, A.M., T.C. Rietveld, A.C. Geurts, and L. Fasotti. Cognitive and graded activity training can alleviate persistent fatigue after stroke: a randomized, controlled trial. Stroke 2012. 43: p. 1046-1051.

125. Adamopoulos, S., J. Parissis, D. Karatzas, et al. Physical training modulates proinflammatory cytokines and the soluble Fas/soluble Fas ligand system in patients with chronic heart failure. J Am Coll Cardiol 2002. 39: p. 653-663.

126. Pedersen, B.K. The anti-inflammatory effect of exercise: its role in diabetes and cardiovascular disease control. Essays Biochem 2006. 42: p. 105-117.

127. Kohut, M.L., D.A. McCann, D.W. Russell, et al. Aerobic exercise, but not flexibility/resistance exercise, reduces serum IL-18, CRP, and IL-6 independent of beta-blockers, BMI, and psychosocial factors in older adults. Brain Behav Immun 2006. 20: p. 201-209.

128. Gao, X.R., C.M. Adhikari, L.Y. Peng, et al. Efficacy of different doses of aspirin in decreasing blood levels of inflammatory markers in patients with cardiovascular metabolic syndrome. J Pharm Pharmacol 2009. 61: p. 1505-1510.

129. Ikonomidis, I., F. Andreotti, E. Economou, et al. Increased proinflammatory cytokines in patients with chronic stable angina and their reduction by aspirin. Circulation 1999. 100: p. 793-798.

130. Yildiz, M., B. Tettenborn, and N. Putzki. Multiple sclerosisassociated fatigue during disease-modifying treatment with natalizumab, interferon-beta and glatiramer acetate. Eur Neurol 2011. 65: p. 231-232. 